\title{
FACTORS INFLUENCING TIME TO DIAGNOSIS AND TREATMENT AMONG PEDIATRIC ONCOLOGY PATIENTS IN KENYA
}

F Njuguna,MD ${ }^{1}$, H Martijn, $\mathrm{BSc}^{2}$, S Langat,BSc ${ }^{1}$, J Musimbi,BSc ${ }^{1}, \quad$ H Muliro ${ }^{1}, \mathrm{BSc}_{\mathrm{S}}, \mathbf{J}$ Skiles, $\mathrm{MD}^{3}$, T Vik, $\mathrm{MD}^{3}$, MN Sitaresmi,MD, $\mathrm{PhD}^{4}$, PM van de Ven,MSc, $\mathrm{PhD}^{5}$, GJL Kaspers, $\mathrm{MD}, \mathrm{PhD}^{2}, \mathbf{S}$ Mostert, $\mathrm{MD}, \mathrm{PhD}^{2}$

${ }^{1}$ Department of Child Health and Pediatrics, Moi University Eldoret, Kenya

${ }^{2}$ Department of Pediatric Oncology-Hematology and Doctor 2 Doctor program, VU University Medical Center, Amsterdam, the Netherlands

3 Department of Pediatrics, Division of Hemato-Oncology, Indiana University School of Medicine, Indianapolis

${ }^{4}$ Department of Pediatrics, Dr Sardjito Hospital, Yogyakarta, Indonesia

5 Department of Epidemiology and Biostatistics, VU University Medical Center, Amsterdam, the Netherlands

Corresponding author: Festus Njuguna, email: muigaifes2000@yahoo.com

Tel. No +254721219761, Box 460630100 Eldoret, Kenya.

Running title: Factors influencing time to diagnosis in oncology

Keywords: childhood cancer, delay, low-income country

This is the author's manuscript of the article published in final edited form as:

Njuguna, F., Martijn, H., Langat, S., Musimbi, J., Muliro, H., Skiles, J., ... Mostert, S. (2016). Factors influencing time to diagnosis and treatment among pediatric oncology patients in Kenya. Pediatric Hematology and Oncology, 33(3), $186-199$. 


\section{ABSTRACT}

. Early diagnosis and start of treatment are fundamental goals in cancer care. This study determines the time lag and the factors that influence the time to diagnosis and start of treatment. Study participants were parents of childhood cancer patients diagnosed between August 2013 and July 2014 in a hospital in Kenya. Patient, physician, diagnosis, treatment, health care system, and total delay were explored using a questionnaire. Demographic and medical data were collected from the patients' medical records. Parents of 99 childhood cancer patients were interviewed (response rate: 80\%). Median total delay was 102 (9-1021) days. Median patient delay (4 days) was significantly shorter than health care system delay (median 87 days; $P<.001$ ). Diagnosis delay (median 94 days) was significantly longer than treatment delay (median 6 days; $P<.001)$. days. Lack of health insurance at diagnosis and use of alternative medicine before attending conventional health services were associated with a significantly longer patient delay $(P=.041$ and $P=.017$, respectively). The type of cancer had a significant effect on treatment delay $(P=.020)$. The type of health facility attended affected only patient delay $(P=$ .03). Gender, age at diagnosis, stage of disease, parents' education level or income, and distance from hospital did not have a significant effect on the length of any type of delay. Training on childhood cancer should be included in the curricula for medical training institutes. In-service workshops should be held for the health workers already working. Families must be obligated to get health insurance. Families should be encourage to attend conventional health facilities and informed on symptoms of cancer through mass media 


\section{INTRODUCTION}

Low and middle-income countries bear the greater burden of childhood malignancies as compared to high-income countries. Of the annual 200,000 cancer cases that occur worldwide in children less than 15 years, about $70-80 \%$ are found in low and middle-income countries. ${ }^{1-4}$

Survival rates in high-income countries are up to $80 \%$, while most countries in Africa have survival rates less than $30 \% .^{5-7}$ This scenario has been attributed to many factors including difficulties in accessing hospital, paucity of trained personnel, lack of investigational and treatment capabilities as well as late presentation. ${ }^{1,2,6,7}$

Early diagnosis of cancer is a fundamental goal in cancer care because it gives opportunity for timely initiation of appropriate treatment, while the disease is at its earliest stages. Early diagnosis and start of treatment may improve prognosis and allow cure to be achieved with minimal side-effects. ${ }^{8}$

Variations in the length of diagnosis and treatment delay may be due to differences in health-care systems as well as patient-related and cancer-related factors. Health-care system factors include access to services, knowledge of providers as well as availability of diagnostic and treatment capabilities. Patient-related factors include age, sex and socio-economic background of the child, while cancer-related factors are mainly related to its clinical presentation and progression. ${ }^{8,9}$

The main purpose of this paper was to assess the various types of delay's seen among pediatric oncology patients at an academic hospital and to determine the factors that influence the time to diagnosis and start of treatment. 


\section{METHODS}

\section{Setting}

Kenya is a low-income country in East-Africa. In 2014 Kenya had an estimated population of 45 million people of which $42 \%$ was aged between $0-14$ years. Half of all Kenyan citizens live below the poverty line.$^{10,11}$

The health system in Kenya is composed of government-owned and private health facilities. These facilities are organized in three levels; primary, secondary, tertiary. Primary health facilities include dispensaries, health-centers and private clinics and are mainly involved in health promotion and prevention and do not have admission facilities. Secondary facilities include mission, private and district hospitals and are able to offer a wide variety of general and some specialized outpatient and inpatient care. Tertiary hospitals are the national referral hospitals and provide sub-specialized services. The majority of population accesses health care by paying out of their pockets. ${ }^{12}$ Health-insurance is provided through private entities or through government-owned National Hospital Insurance Fund (NHIF). All persons working in the formal sector contribute to NHIF while those in the informal sector can join voluntarily at a cost of about 25 US dollars per year which covers inpatient care for the nuclear family in governmentowned facilities. ${ }^{13}$

The study was conducted at Moi Teaching and Referral Hospital (MTRH) in Eldoret, Western Kenya. MTRH has a service area of 16-18 million people, which is about $40-45 \%$ of all inhabitants of Kenya. Although an estimated 700 childhood cancer patients under 15 years of age are expected in the service area of MTRH, only 100-110 children are annually diagnosed with 
cancer. ${ }^{14}$ MTRH has a bed capacity of 700 patients of which 72 beds are located on the pediatric ward. Twelve of the 72 beds are reserved for oncology patients.

\section{Study design}

This cross-sectional study was conducted using a semi-structured questionnaire. The focus was to assess the various types of delay among pediatric oncology patients seen at MTRH and to determine the factors that influence the time to diagnosis and start of treatment. Participants were parents of childhood cancer patients who were newly diagnosed between August 2013 and July 2014. We interviewed parents of all consecutive patients.

By definition, "patient delay" refers to time from onset of symptoms to first time patient has contact with conventional health-care provider regarding symptoms attributable to cancer. "Health-care system delay" refers to time from first presentation to health-care provider to initiation of treatment. "Diagnosis delay" represents time from onset of symptoms to time the diagnosis of cancer is confirmed. "Treatment delay" refers to time from confirmation of diagnosis to start of treatment. "Physician delay" refers to time from first contact with healthcare provider to confirmation of diagnosis. "Total delay" refers to time from onset of symptoms to initiation of therapy (Figure1). ${ }^{8}$

Parents were asked when symptoms started and on which date they attended the first health-care provider. If parents could not recall the exact date of onset of symptoms and/or date of first contact with health-care provider, preconceived categories were used: early-mid-end of the month. During analysis we transformed these categories into day 5 - day 15 - day 25 (respectively) of the month. 
The questionnaire contained open and structured questions that parents could evaluate on two to five-point rating scales. The items in the questionnaire had been derived from extensive literature studies. Questionnaire was pilot-tested for its content, clarity and cultural sensitivities on parents of 5 children and revised accordingly. Demographic and medical data was collected from medical records of childhood cancer patients: diagnosis, date of diagnosis, age at diagnosis, stage of disease and date of start treatment. Respondents rendered informed consent. Anonymity and confidentiality were guaranteed. Study was approved by Research Ethics Committee of MTRH.

\section{Data Analysis}

Data management and analysis were executed by using SPSS. For each variable frequency distribution, median, means and standard deviations were calculated. Kruskal-Wallis test and Mann-Whitney test were used to compare time lag between different categorical variables. To compare paired time lag between patient delay and health-care system delay and between diagnosis delay and treatment delay, Wilcoxon Signed-Rank test was used. Statistical significance was set at $\mathrm{p}<0.05$.

\section{RESULTS}

\section{Demographic characteristics}

Between August 2013 and July 2014, 123 children were newly diagnosed with a malignancy. Caretakers of 99 children (response rate 80\%) were interviewed. Parents of 21 patients (17\%) were not interviewed for different reasons: patient deceased before interview could take place (52\%), abandonment of treatment (24\%), language barrier as parents spoke a rare dialect (14\%), refusal to participate $(10 \%)$. Three patients $(2 \%)$ had to be excluded from analysis because parents could not recall when symptoms started or what the date was of first health facility visit. 
Respondents included following caretakers: mothers (56\%), fathers $(21 \%)$, uncles/aunts $(10 \%)$, grandparents $(6 \%)$, siblings $(4 \%)$, both parents $(2 \%)$, guardian (1\%). Table 1 presents parents' characteristics.

Of 99 children, $67 \%$ were male. Patients' age at diagnosis was a mean of 6.5 years $(\mathrm{SD}=3.8)$ and median 5.7 years. Hematological tumors contributed for $54 \%$ and solid tumors for $46 \%$ of all cases. There were no significant differences in patients' age at diagnosis, gender or type of cancer between respondents $(\mathrm{n}=99)$ and non-respondents $(\mathrm{n}=24)$.

\section{Various types of delay}

Table 2 presents socio-demographic and clinical characteristics and the various types of delay. Some parents could not tell the exact date of onset of symptoms $(66 \%)$ or the exact date of first contact with a conventional health-care provider $(55 \%)$ and therefore used the preconceived categories. Paired comparison of patient delay and health-care system delay showed the latter to be significantly longer $(\mathrm{P}<0.001)$. Paired comparison of diagnosis delay and treatment delay showed the first to be significantly longer $(\mathrm{P}<0.001)$.

\section{Onset of symptoms}

Children suffered from following major symptoms before seeking medical care: swelling (51\%), pain (35\%), fever (26\%), vomiting (15\%), loss of weight (13\%), loss of appetite (10\%), fatigue (8\%), weakness $(7 \%)$. After noticing these symptoms parents sought advice from relatives and friends (50\%), and decided to first wait and watch (36\%). Table 2 shows patient delay.

\section{Distance and transportation to hospital}


Of all participants, $76 \%$ lived more than $100 \mathrm{~km}$ from MTRH. Travel time to MTRH was: $<1$ hour (4\%), 1-3 hours (41\%) and $>3$ hours (55\%). Mode of transportation to reach MTRH is: public transport (94\%), motorbike (23\%), walking (18\%), private car $(5 \%)$, renting motorbike $(4 \%)$, ambulance $(2 \%)$ and renting vehicle $(1 \%)$. Note that some respondents reported a combination of transportation modes. Travelling to hospital is considered to be: expensive (81\%), time consuming (74\%) and difficult (50\%). No significant difference was found between distance to MTRH and any type of delay (Table 2).

\section{Financial situation}

Minority (38\%) of households have regular income. Main economic provider is: father (48\%), both parents $(25 \%)$, mother $(20 \%)$, grandparents $(5 \%)$, aunt $(1 \%)$ and relatives $(1 \%)$. Families lived either below (37\%) or above (63\%) poverty line of 1.25 USD per day. Table 2 illustrates that no statistically significant difference in all types of delay was found between families living below or above poverty line. When coming to MTRH, 71 families (72\%) lose daily wages and 40 families (41\%) lose profits from farming the land.

\section{Health-insurance}

Most families (67\%) did not have health-insurance before onset of illness and 64\% did not have insurance when they first came to MTRH. Having health-insurance at diagnosis resulted in a significantly less patient delay $(\mathrm{P}=0.049)$ as shown in Table 2 .

\section{Social support for alternative and conventional treatment}

Parents (58\%) were advised to seek alternative treatment for disease in their child by: grandparents (53\%), village community (51\%), friends $(47 \%)$, relatives $(42 \%)$, spouse $(26 \%)$, 
religious community (16\%), medical personnel $(7 \%)$ and clan elder (2\%). Parents (34\%) were advised not to attend public hospitals by: village community (50\%), relatives (44\%), friends $(41 \%)$, grandparents (32\%), spouse (24\%), religious community $(9 \%)$.

\section{Alternative treatment}

After noticing symptoms of the child, 58 parents (59\%) sought alternative treatment for their children: praying ceremonies (41\%), visiting herbalist (36\%), special food intake (11\%), and attending traditional healer (3\%). Reasons for using alternative treatment are illustrated in Table

3. Use of alternative medicine resulted in significantly longer patient delay $(\mathrm{P}=0.017)$, diagnosis delay $(\mathrm{P}=0.030)$ and total delay $(\mathrm{P}=0.033)$.

\section{First contact with conventional health-care facilities}

Patients' first contact with a health-care facility was at primary- $(60 \%)$, secondary- $(38 \%)$ and tertiary-care level (2\%). During this first contact, patients were helped by doctor $(68 \%)$ or nurse $(32 \%)$. Most children (69\%) were initially treated for one or more other illnesses: e.g. malaria (34\%), infection (25\%), pain (15\%) and anemia (7\%).

\section{Cancer education during first contact with conventional health-care facilities}

During first contact with conventional health-care provider cancer was mentioned as possible diagnosis to 12 parents $(12 \%)$. This health-care provider subsequently encouraged parents to seek conventional cancer treatment $(92 \%)$ and discouraged complementary alternative treatment (42\%). Curability of cancer was discussed with 10 of these parents (83\%): $90 \%$ were told that cancer is curable and $10 \%$ that cancer is incurable. Affordability of cancer treatment was discussed with 4 of these parents (33\%): 3 (75\%) were told cancer treatment is not affordable 
and $1(25 \%)$ that cancer treatment is affordable. During first contact with conventional healthcare provider $20 \%$ of all parents were advised to enroll in NHIF.

\section{Total number of visits to conventional health-care facilities}

Table 4 illustrates type of health-care facilities patients attended prior to receiving cancer treatment at MTRH. Total number of visits to other conventional health-care facilities prior to attending MTRH ranged from 1 to 9 visits with a mean of 3 visits $(\mathrm{SD}=1.5)$, and median of 3 visits. The first health facility attended significantly affected patient delay $(\mathrm{P}=0.03)$, but not other forms of delay.

\section{Health beliefs}

In total 81 parents $(82 \%)$ had heard of cancer before their child had been diagnosed with cancer. These parents perceived cancer to be curable $(61 \%)$, incurable $(37 \%)$ or were uncertain $(3 \%)$.

Prior to coming to MTRH conventional cancer treatment was thought to be unaffordable (78\%), affordable $(11 \%)$, or parents were unsure $(11 \%)$. Use of surgery in cancer treatment was believed to be: helpful $(96 \%)$, necessary $(83 \%)$, frightening $(62 \%)$, causing death $(46 \%)$ and spreading cancer $(32 \%)$.

\section{Reasons for delay in coming to MTRH}

Many families (97\%) encountered difficulties coming to MTRH for the first time. Table 5 illustrates reasons for delay in attending MTRH. Before coming to MTRH, 25 families (25\%) had heard of not being allowed to take your child home in public hospitals in case families are unable to pay their medical bills (hospital detention practices). Ten of these families (40\%) 
delayed coming to MTRH because of this. Of all 99 parents, $86 \%$ stated that parents would come sooner to public hospitals if children were not detained over unpaid medical bills.

\section{First contact with MTRH}

Before attending MTRH for the first time, 23 children (23\%) had already been diagnosed with cancer at primary- $(9 \%)$, secondary- $(83 \%)$ and tertiary-care level $(9 \%)$. The first department at MTRH which parents attended was: sick child clinic (77\%), eye clinic (9\%), hemato-oncology clinic (7\%), ear nose and throat clinic (3\%), private wing outpatient clinic $(2 \%)$, casualty clinic (2\%) and dental clinic (1\%). Most children (99\%) were immediately admitted and investigated for cancer.

\section{DISCUSSION}

This study demonstrates that diagnosis and treatment delay is a major issue. Median total delay was 102 days. Overall patient delay (median 4 days) was considerably shorter than health-care system delay (median 87 days). Although the total delay figures are similar to findings in Nigeria where median total delay was 109 days, our figures are longer than what has been reported in other studies done in Africa. ${ }^{15-18}$ A study done in Western Kenya and Uganda, specifically looking at Burkitt lymphoma, reported total delays of 12.1 and 12.9 weeks respectively. This is shorter than what we found though close to what we found in our Non-Hodgkin lymphoma patients who had a mean total delay of 73 days. ${ }^{19}$ Similar studies in Egypt and South-Africa reported median total delays of 47 and 34 days respectively. The short total delay reported from South-Africa could be a reflection of the superior infrastructure and health-care system in the country and is similar to total delays found in high-income countries, such as Canada which also has a median total delay of 34 days..$^{15-18}$ 
Health-care system delay in our study was significantly longer than patient delay. This is unlike what has been reported by the Nigerian study where patient delay was longer than health-care system delay as well as the Burkitt lymphoma study in Western Kenya. However the Egyptian and South-African study also demonstrated a longer health-care system delay. ${ }^{15-19}$ This may mean that parents in our setting come to hospital relatively fast but a lot of delays occur in the health-care system. These could be because medical workers are not able to suspect and make the right diagnosis. It could also reflect complex referral systems as well as unavailability and inefficiencies of equipment and personnel to make correct diagnosis. Dual physician practices and absenteeism could also be contributing factors. ${ }^{20-21}$ Government should enforce policies to stop dual physician practices by raising government salaries and obligating doctors to work fulltime in public hospitals. Training on how to suspect and diagnose cancer among health-care workers could help to reduce the health-care system delay.

The treatment delay was a median of 6 days. This is similar to what was reported in Nigeria. ${ }^{15}$ This delay could especially occur among patients who are diagnosed in other hospitals and are referred to MTRH for care. It could also happen as some families take time before reaching a decision on whether to start treatment for their children. Some patients may be quite sick and supportive measures are undertaken before their treatment is initiated. The lack of consistent availability of senior doctors also slows down the process as their opinion may be needed before treatment is started. ${ }^{20-21}$

This study did not find a significant difference in the delays between the two genders. The sex of the child has been found not to influence diagnosis delay in different studies. ${ }^{16,17,23,24}$ This may 
imply that there are no differences in health-seeking behavior regardless of the gender of the child.

The longest total delay was for the age group 6-10 years though it was not significantly longer when compared to the other age groups. Those who were less than 5 years had the shortest delay which could be because the common tumors in this age group are aggressive and may lead to faster presentation. Most studies have reported the time lag to be longer for older children. However in Nigeria there were no differences among the age groups ${ }^{15,16,17}$

Most common diagnosis in this series was nephroblastoma, followed by non-Hodgkin lymphoma and acute lymphoblastic leukemia. The type of cancer only significantly affected treatment delay. However, it is important to note that retinoblastoma patients had the longest median patient delay (30 days) as well as very long median physician delay (109 days). This could imply that signs and symptoms of retinoblastoma are not easily recognized by parents and health-care providers.

The Nigerian study found long patient and physician delays among retinoblastoma and Hodgkin lymphoma patients. ${ }^{15}$ Hodgkin lymphoma commonly presents in an indolent manner with painless adenopathy which mimics tuberculosis of lymphatic system. Given the high prevalence of tuberculosis in Low income settings, most of these patients are likely to be treated with antituberculosis medication and an alternative diagnosis is only sought when there is no response several months later. ${ }^{25-26}$ However, our median patient and physician delays for Hodgkin lymphoma patients were not extremely long compared to other types of cancers.

Treatment delay was longest for Kaposi's sarcoma, although one needs to acknowledge that there were only two patients. Most patients in this set-up have Human Immunodeficiency virus 
associated Kaposi sarcoma. Unless the disease is life threatening the specific treatment for Kaposi sarcoma is delayed and antiretrovirals are used to improve the patients' immunity and reduce the Kaposi sarcoma burden. This may lead to the long treatment delay documented here. ${ }^{27}$

The shortest total delays were among Non-Hodgkin lymphoma and acute myeloid leukemia patients. They also had shortest patient delays. This could be attributed to the nature of diseases whereby Non-Hodgkin lymphoma usually presents with rapidly enlarging mass and acute myeloid leukemia presents with significant bleeding. Other authors have reported a shorter total delay for acute leukemia compared to solid tumors. ${ }^{21,22}$

Only $33 \%$ of patients had health-insurance (NHIF) at time of onset of symptoms. ${ }^{13}$ Having insurance resulted in significantly shorter patient delay compared to those without. This is one of the areas that the government and other stakeholders should focus on given that we have previously shown that having insurance also reduces risk of abandonment and improves survival. 28,29

More than half of families reported use of alternative treatment before coming to MTRH. Use of alternative treatment resulted in significantly longer patient delay as well as diagnosis delay. Previous studies in this setting have shown that many families use complementary alternative medicine. ${ }^{29}$ While parents had valid reasons for using alternative treatment it shows that time is lost before they seek care in a conventional health facility. More community education would alleviate this though the challenges associated with accessing health-care in Kenya need to be addressed as well. These include long distances to facilities, poor facilities as well as the negative perceptions about poor service provision from health-care workers. ${ }^{31-32}$ 
Parents' level of education did not have a significant effect on either patient or total delay. Other studies have given contrasting reports. In Egypt shorter total delay among parents with higher education level was reported. ${ }^{17}$ Studies in Nigeria and South-Africa however showed that level of education has no effect on total delays. The level of income also did not have an effect on the delays. Majority of families lived more than $100 \mathrm{~km}$ from MTRH but this had no effect on either patient or total delays. The cost of travel to hospital would be expected to be an economic burden to most poor families. All these socio-economic factors are interrelated and did not have a significant effect on delay patterns.

Hospital detention practices are not known to the majority of parents prior to coming to MTRH. Among those who were aware $40 \%$ reported it as a reason to delay coming to hospital and $36 \%$ as a reason to seek alternative treatment. Hospital detention occurs when families are not allowed to take their child home after discharge due to failure to pay hospital bills. This has been shown to have negative consequences on the families and may influence their health-seeking behaviour. The families feel desperate, powerless and feel the hospital is like a prison. Families may avoid coming to the hospital when they are aware of the detention practices. ${ }^{30,33,34}$ It may contribute to the lower reported than expected childhood cancer incidence (100-110 children versus 700 children) in the service area of MTRH. ${ }^{14}$

In conclusion we found that total delay is much longer than has been reported in most previous studies. The only factor that significantly affected total delay was use of alternative treatment. The health-care system delay was significantly longer than patient delay. Factors associated with long patient delay include: health-insurance at onset of diagnosis and use of alternative treatment. There were no factors that significantly affected physicians' delay as well as healthcare system delay. Treatment delay was only significantly associated with the type of cancer. 
This study had several limitations. There could have been a recall bias regarding the onset of symptoms and health facility attendance. Parents may also have been giving socially desirable answers as criticism is not encouraged in the Kenyan culture. ${ }^{30}$

Based on our study findings, we recommend that training on childhood cancer be incorporated into the curricula of medical training institutions. Workshops to sensitize those already working should be organized as well as presentations in scientific conferences of various cadres of health workers Sensitization of lay communities through mass media on common symptoms of cancer should be organized through the division of health promotion at the Ministry of Health. The public also needs to be encouraged to visit conventional health facilities first whenever their children are unwell. The government should make it mandatory to register with NHIF when acquiring the national identity card at the age of 18 years as well as when children get their birth certificates.

ACKNOWLEDGEMENTS We wish to acknowledge Doctor 2 Doctor for their logistical support. The financial support for this study was from a SIOP fellowship.

\section{DECLARATION OF INTEREST}

All the authors have no conflicts of interest to declare. 


\section{REFERENCES}

1. Hadley LG, Rouma BS, Saad-Eldin. Challenge of paediatric oncology in Africa Semin Pediatr Surg 2012;21:136-41

2. Harif M, Traoré F, Hessissen L, et al. Challenges for paediatric oncology in Africa Lancet Oncol 2013;14:279-81

3. Hessissen L, Madani A. Paediatric oncology in Morocco: achievements and challenges. J Pediatr Hematol Oncol 2012;34: 21-2

4. Israels T, Ribeiro RC, Molyneux EM. Strategies to improve care for children with cancer in Sub-Saharan Africa. Eur J Cancer 2010;46:1960-6

5. Yao JJ, Couitchere L, Atimere Y, et al. Childhood cancer in Côte d'Ivoire, 1995 2004: challenges and hopes. S Afr Med J 2012; 8; 113-5

6. Slone JS, Chunda-Liyoka C, Perez M, et al. Paediatric malignancies, treatment outcomes and abandonment of paediatric cancer in Zambia. PLoS One 2014; 21;9 
7. Mostert S, Njuguna F, Kemps L et al. Epidemiology of diagnosed childhood cancer in Western Kenya. Arch Dis Child 2012;97:508-12

8. Dang-Tan T, Franco EL. Diagnosis delays in childhood cancer: a review. Cancer 2007;110:703-13.

9. Brasme JF, Morfouace M, Grill J, et al. Delays in diagnosis of paediatric cancers: a systematic review and comparison with expert testimony in lawsuits. Lancet 2012;13:445-59.

10. Kenya population situation analysis. Available at: http://countryoffice.unfpa.org/kenya/drive/FINALPSAREPORT. (Accessed October $13,2014)$

11. Kenya population 2014. Available at: http://worldpopulationreview.com/countries/kenya-population/ (Accessed October $13,2014)$

12. Muga R, Kizito P, Mbayah M. Overview of the health system in Kenya Available at: http://dhsprogram.com/pubs/pdf/spa8/02chapter2.pdf (Accessed October 23, 2014)

13. National hospital insurance fund. Available at: http://www.nhif.or.ke (Accessed October 14, 2014) 
14. Njuguna F, Mostert S, Slot A, et al. Abandonment of childhood cancer treatment in Western Kenya. Arch Dis Child 2014;99:609-14

15. Chukwu BF, Ezenwosu OU, Ikefuna AN, et al. Diagnostic Delay in Pediatric Cancer in Enugu, Nigeria: A Prospective Study Pediatr Hematol Oncol 2015;32:16471

16. Stefan DC, Siemonsma F. Delay and causes of delay in the diagnosis of childhood cancer in Africa. Pediatr Blood Cancer 2011;56:80-5

17. Abdelkhalek E, Sherief L, Kamal N, et al. Factors associated with delayed cancer diagnosis in Egyptian children Clin Med Insights Pediatr 2014;4:39-44

18. Dang-Tan T1, Trottier H, Mery LS et al. Delays in diagnosis and treatment among children and adolescents with cancer in Canada. Pediatric Blood Cancer 2008;51:468-74

19. Geoffrey CB, Jennifer PC, Peter OS et. al Factors influencing time to diagnosis and initiation of treatment of endemic Burkitt Lymphoma among children in Uganda and western Kenya: a cross-sectional survey Infect Agent Cancer. 2013; 8: 36. 
20. Johannessen KAI, Hagen TP. Physicians' engagement in dual practices and the effects on labor supply in public hospitals: results from a register-based study. BMC Health Serv Res $2014 ; 14: 299$

21. Russo G, McPake B, Fronteira I, Ferrinho P. Negotiating markets for health: an exploration of physicians' engagement in dual practice in three African capital cities. Health Policy Plan 2014;29:774-83

22. Russo G1, de Sousa B, Sidat M, et al. Why do some physicians in Portuguesespeaking African countries work exclusively for the private sector? Findings from a mixed-methods study. Hum Resour Health 2014;12:51

23. Brown BJ, Ajayi SO, Ogun OA, et al. Factors influencing time to diagnosis of childhood cancer in Ibadan, Nigeria. Afr Health Sci 2009;9:247-53

24. Araz NC, Guler E. Delays in Diagnosis of Childhood Cancer in Southeastern Turkey and the Associated Factors. Pediatr Hematol Oncol 2015;32:153-163

25. Law MF, Ng TY, Chan HN, et al. Clinical features and treatment outcomes of Hodgkin's lymphoma in Hong Kong Chinese. Arch Med Sci 2014;10:498-504

26. Ahmed HG, Nassar AS, Ginawi I. Screening for tuberculosis and its histological pattern in patients with enlarged lymph node. Patholog Res Int 2011:417635 
27. La Ferla L, Pinzone MR, Nunnari G, et al. Kaposi' s sarcoma in HIV-positive patients: the state of art in the HAART-era. Eur Rev Med Pharmacol Sci $2013 ; 17: 2354-65$.

28. Axt J, Abdallah F, Axt M, et al. Wilms tumor survival in Kenya. J Pediatr Surg 2013;48:1254-6.

29. Mostert S, Njuguna F, van de Ven PM, et al. Influence of health-insurance access and hospital retention policies on childhood cancer treatment in Kenya. Pediatr Blood Cancer 2014;61:913-8

30. Njuguna F, Mostert S, Seijjfert A, et.al. Parental experiences of childhood cancer treatment in Kenya. Support care cancer 2015;23:1251-9

31. Chuma J, Okungu V Viewing the Kenyan health system through an equity lens: implications for universal coverage. Int J Equity Health 2011;26:10:22

32. Echoka E, Makokha A, Dubourg D,et al. Barriers to emergency obstetric care services: accounts of survivors of life threatening obstetric complications in Malindi District, Kenya. Pan Afr Med J 2014;17 
33. Mostert S, Njuguna F, Langat S. et al. Two overlooked contributors to abandonment of childhood cancer treatment in Kenya: parents' social network and experiences with hospital retention policies. Psychooncology 2014;23:700-7

34. Mostert S. Lam C. Njuguna F. et al. Hospital detention practices: statement of a global taskforce. The Lancet 2015:386:649 